\title{
Experience in modeling a comfortable environment in the transport compartment
}

\author{
Vladimir Pankratov ${ }^{1}$, Alexey Golikov ${ }^{1}$, Elena Pankratova $^{1}$, Marina Barulina $^{* 1}$, and Sofiya \\ Galkina $^{1}$ \\ ${ }^{1}$ Institute of Precision Mechanics and Control, Russian Academy of Sciences, 410028, 24 Rabochaya \\ st., Saratov, Russia
}

\begin{abstract}
The theoretical base was developed and the mathematical model of dynamic thermal processes in a compartment of public transport was constructed. The software which is realized the constructed mathematical model was developed. The mathematical model provides for the possibility of taking into account the most possible environmental conditions which can have place in the actual operating conditions of a transport even the angle of illumination - the current temperature of the external environment, the presence of solar radiation taking into account the angle of sun's illumination, and the temperature of the roadway. The software allows calculation and visualization non -stationary temperature fields in the public transport's compartments using the example of a trolley bus. For a specific trolley bus design, a series of experiments to calculate the comfortable temperature for passengers were conducted, These experiments are showed the performance of the constructed model and allowed to formulate a specific proposal for improving the thermoregulation system of the trolleybus' passenger compartment.
\end{abstract}

\section{Problem and theoretical base}

The necessity to design of comfortable public transport considering heat distribution, inner heat sources, compartment ventilation, and other factors requires the knowledge of the nature and parameters of convection flows and thermal processes occurring in the compartment. Otherwise, the complexity of heat exchange processes [1-9] makes it necessary to apply a wide range of theoretical and experimental methods and tools to study such processes.

The purpose of this work is to create and to study a mathematical model of interconnected nonstationary heat-mass-exchange processes in public transport's compartment on the example of the trolleybus' passenger compartment.

For achieving the goal, the following tasks must be solved:

- to construct the equations of heat-mass-exchange processes which have the place in the trolleybus' passenger compartment, taking into account the thermal situation outside the

\footnotetext{
*Corresponding author: marina@barulina.ru
} 
trolleybus, the compartment thermoregulation system, amount of passengers inside the compartment, the number of incoming and outgoing passengers;

- to determine and visualize inhomogeneous, three-dimensional, nonstationary temperature fields and their expansion over the compartment space by modern computer tools;

The authors have rich experience in studying heat exchange processes in complex devices and aerospace instrumentation systems [1-4]. This experience can be used for the developing methods and research tools to solve the main purpose set in this work.

The presence of different types of heat exchange inside the trolleybus' passenger compartment and the conditions of heat exchange with the external environment leads to the complex Cauchy and Dirichlet boundary value problem, which can't be solved by using the traditional precise analytic methods used in heat transfer problem [5,7]. Also, it is not possible to solve this problem using approximate analytical methods $[5,7]$ due to the high complexity of thermal processes and calculation of the temperature field model. So we can solve this problem by numerical methods only and appropriate algorithms by using computer technology.

\section{Mathematical model and constitutive relations}

The basis of the proposed calculation method is a generalization of the method "elementary" balances. The choice of the method conditioned by the complexity of the set temperature problems, variety, and interconnection of the physical effects and processes, which take place in the considered object.

The proposed method of the calculation of the thermal fields allows taking into consideration the most important types of heat transfer that take place in the trolleybus' passenger compartment. In using that method the trolleybus' inner space and all elements in it are divided into the structural elements connected to each other by thermal relations. Then the average temperature in the $i$-th volume and at the time $t+\Delta t$ can be calculated by the following equation [8]:

$$
T_{i}(t+\Delta t)=\left[1-\frac{\Delta t}{c_{i}}\left(\sum_{j=1}^{N} q_{i j}+q_{i c}\right)\right] T_{i}+\frac{\Delta t}{c_{i}}\left(\sum_{j=1}^{N} q_{i j} T_{j}+q_{i c} T_{c i}+Q_{i}\right),
$$

where: $T_{i}(t), T_{i}(t+\Delta t), c_{i}(i=1, \ldots, M)$ - temperatures of the $i$-th elements at the present and subsequent moment and its heat capacity; $q_{i j}$ - thermal characteristic of a connection between elements $i, j(j=1, \ldots, N) ; q_{i c}$ - thermal characteristic of a connection between $i$-th element and the environment; $T_{\mathrm{c} i} i^{-}$environment temperature; $Q_{i^{-}}$heat source power; $M-$ number of elements; $N$ - the number of elements with heat contact with $i$-th element; $\Delta t$ the calculation step.

Equation (1) is an explicit one-step difference scheme and can be implemented in software simple enough. The thermal conductivity q, included in (1), is calculated on the base of the heat and mass transfer laws of Fourier, Newton, Stefan-Boltzmann, the criteria equations of Nusselt, Reynolds, Grashof, Prandtl, the theory of similarity of aerodynamic, electrical and thermal processes and experimental studies. That makes possible to study with difference scheme (1) thermal processes in devices under various conditions, including vacuum, low pressure, gravity, and the influence of the various type of emission, that leads to heating.

The value of the total thermal conductivity, characterized by the thermal characteristic $q$ included in (1), can be represented as:

$$
q=q_{T}+q_{K}+q_{I}=\alpha_{T} f_{T}+\alpha_{K} f_{K}+\alpha_{I} f_{I},
$$

where $q_{T}, q_{K}, q_{I}$ - thermal characteristics, which include heat exchange by thermal conductivity, convection, and radiation respectively; $\alpha_{T}, \alpha_{K}, \alpha_{\mathrm{U}}$ - functions of geometrical and thermophysical parameters of the elementary volumes; $f_{\mathrm{T}}, f_{\mathrm{K}}, f_{\mathrm{U}}$ - functions of the temperature of the elementary volumes. 
The components of the thermal characteristics for different types of heat transfer included in (2) can be calculated using the following formulas:

Heat exchange by thermal conductivity (conduction).

The formula for calculating the thermal characteristic for a flat multilayer side has the form [1]:

$$
q_{T}=S / \sum_{i=1}^{n} \frac{\ell_{i}}{\lambda_{i}}
$$

where $S$ - area of the normal for heat flow surface; $l_{i}$ - the thickness of the $i$-th layer; $\lambda_{i}$ - thermal conductivity coefficient of the $i$-th layer.

Heat transfer by natural (free) and forced convection.

The general formula for calculating thermal characteristic in that case is:

$$
q_{K}=\alpha_{K} S
$$

where $\alpha_{K}$ - heat emission coefficient; $S$ - square of the heat emission surface.

The heat transfer coefficient $\alpha_{K}$ are determined on depends on heat transfer laws that determine the modes of movement of the environment.

Heat exchange by radiation.

In that case, thermal conductivity coefficients have the next structure:

$$
q_{h}=\alpha_{H} S \text {. }
$$

where $\alpha_{И}=5.67 \cdot 10^{-8} \varepsilon_{\Pi}\left[\left(T_{1}+273\right)^{2}+\left(T_{2}+273\right)^{2}\right]+\left(T_{1}+T_{2}+546\right)^{2}$, $\varepsilon_{\Pi}=\varepsilon_{1} \varepsilon_{2} /\left(\varepsilon_{1}+\varepsilon_{2}+\varepsilon_{1} \varepsilon_{2}\right)$ - reduced power of blackness of the bodies surfaces between which heat transfer occurs; $0<\varepsilon_{1}, \varepsilon_{2}<1$ - powers of the bodies surface blackness; $T_{1}, T_{2}$ - bodies surfaces temperatures in ${ }^{o} \mathrm{C}$.

The mathematical model provides for the possibility of taking into account all possible environmental conditions which can have place in the actual operation conditions of a given vehicle - the current temperature of the external environment, the presence of solar radiation taking into account the angle of illumination, the temperature of the roadway, traffic conditions along the route, time and frequency of stops, occupancy of passengers and other factors.

\section{Software and numerical results}

The constructed model of temperature fields was implemented in special software. The developed software allows visualization of temperature changes in the trolleybus' passenger compartment in dynamics depends on the specified conditions of the environmental and inner perturbing factors. The calculated temperature fields using the mathematical model based on formulas (1)-(5), shown in Fig.1 - Fig.8.

The temperature fields in Fig. 1 - Fig. 4 were obtained at conditions that simulated a winter day. Ambient temperature is negative and equal to $-15^{\circ} \mathrm{C}$, all heatings are turned on, ventilation of the passenger compartment is partly turned on, and the passenger compartment is full up to $100 \%$ (Fig.1, Fig.3) and 50\% (Fig.2, Fig.4). The maximum temperature takes place in the zone of location of the heaters $\left(36^{\circ} \mathrm{C}\right)$ - these zones are clearly visible in Fig.3.

The result of calculating the temperature fields in the hot summer day is shown in Fig.5 - Fig.8. Ambient temperature is equal to $35^{\circ} \mathrm{C}$, ventilation of the passenger compartment is fully turned on, all windows and hatches are fully opened, and the compartment fullness is $100 \%$ (Fig.5, Fig.7) and 50\% ((Fig.6, Fig.8) occupied by passengers. 


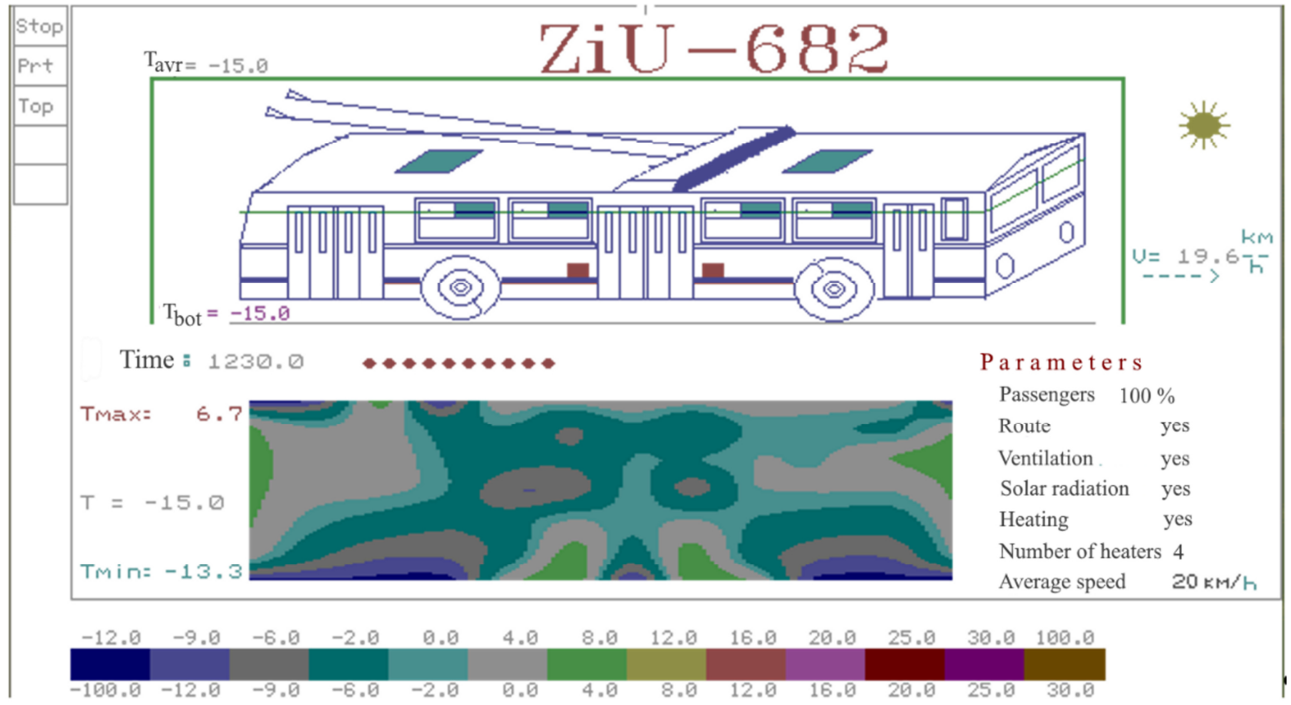

Fig. 1. The temperature field at the level of the ceiling of the trolleybus' passenger compartment in winter. The passenger compartment fullness $100 \%$.

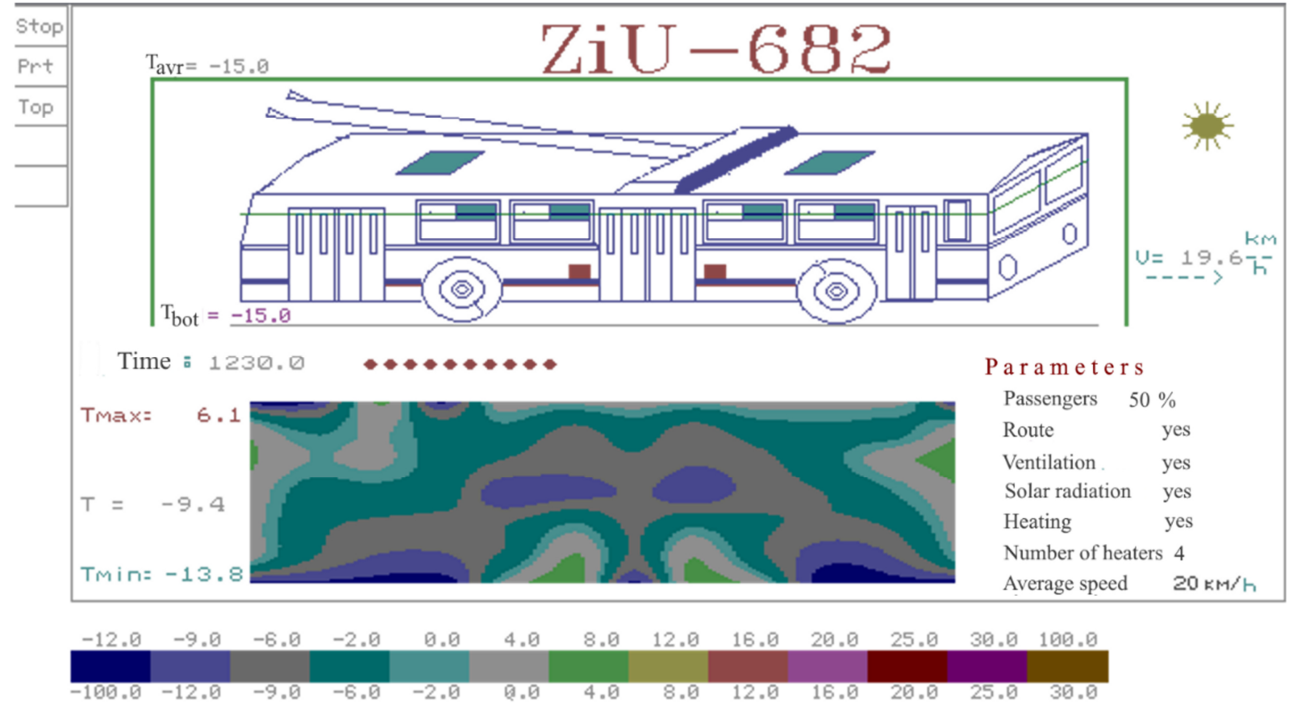

Fig. 2. The temperature field at the level of the ceiling of the trolleybus' passenger compartment in winter. The passenger compartment fullness $50 \%$. 


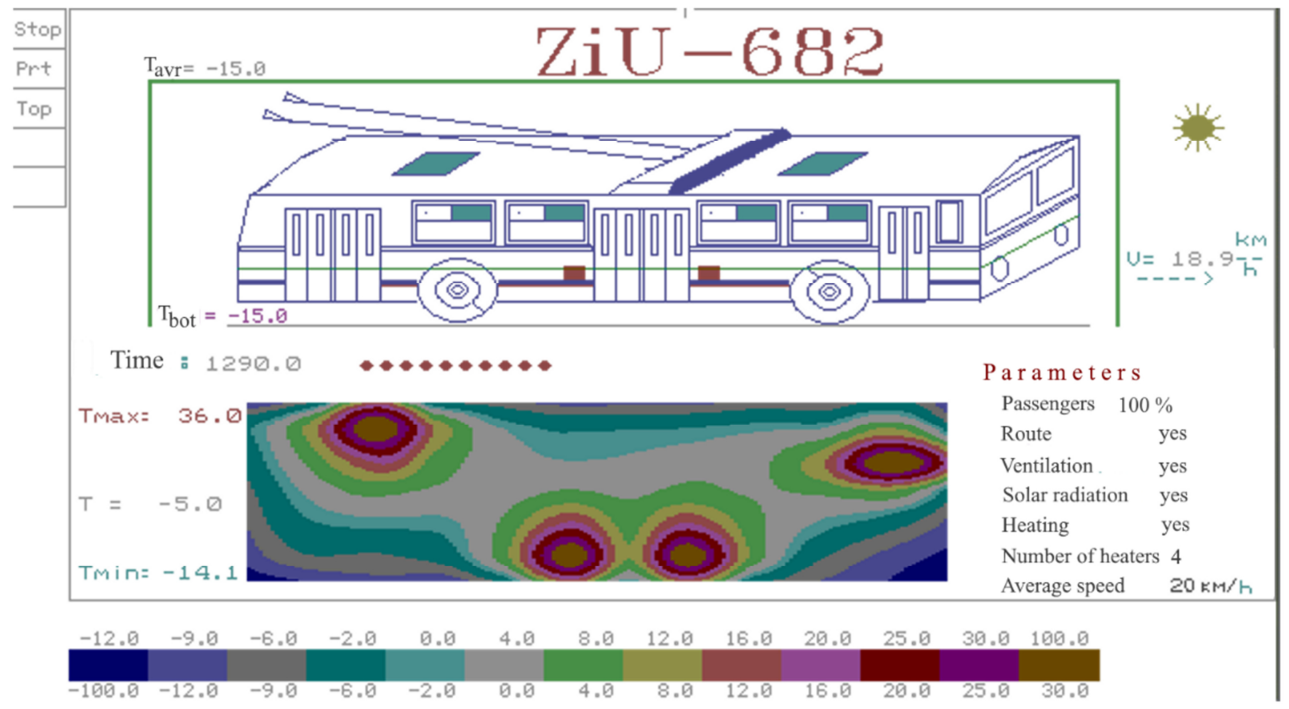

Fig. 3. The temperature field at the level of the sitting places in winter. The passenger compartment fullness $100 \%$.

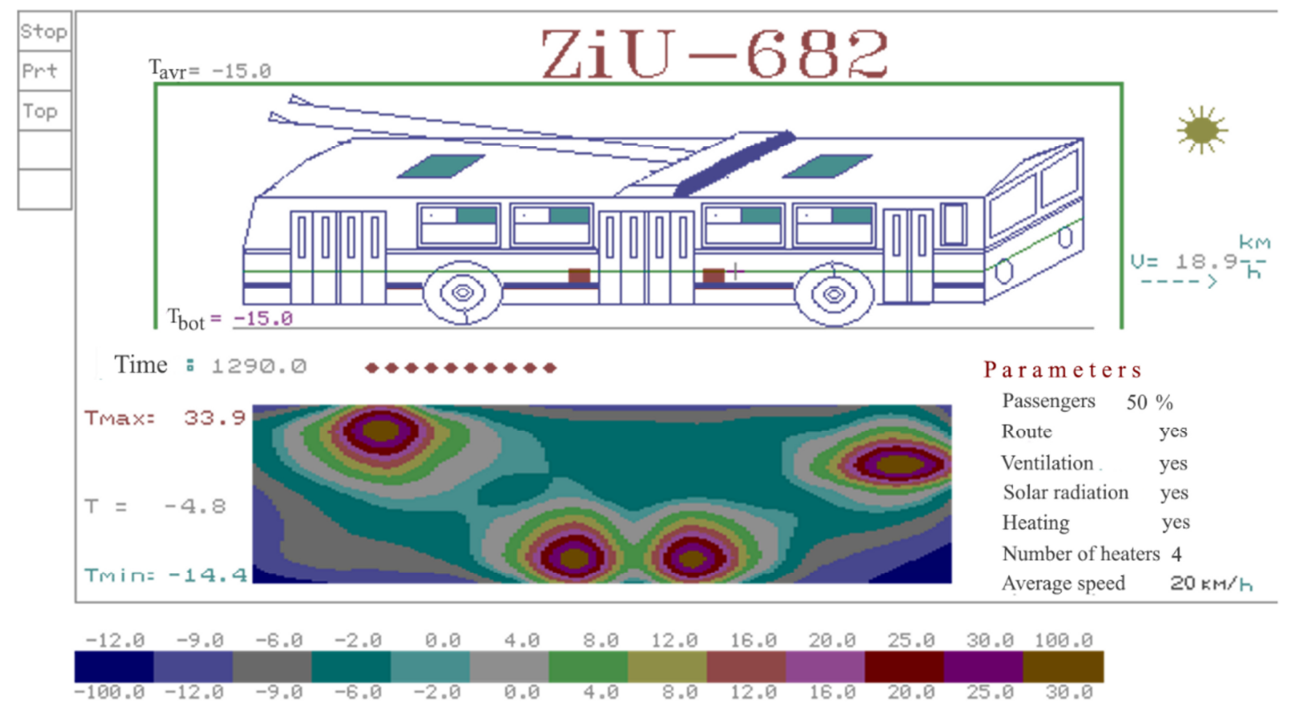

Fig. 4. The temperature field at the level of the sitting places in winter. The passenger compartment fullness $50 \%$. 

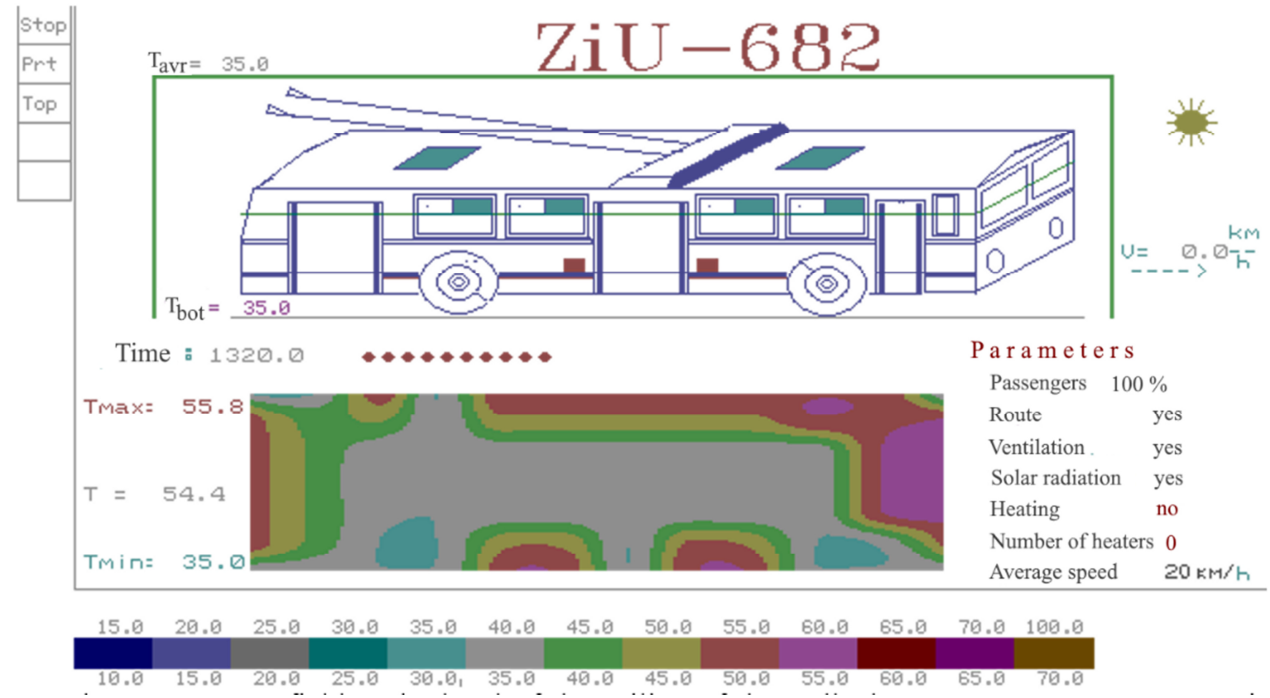

Fig. 5. The temperature field at the level of the ceiling of the trolleybus' passenger compartment in summer. The passenger compartment fullness $100 \%$.

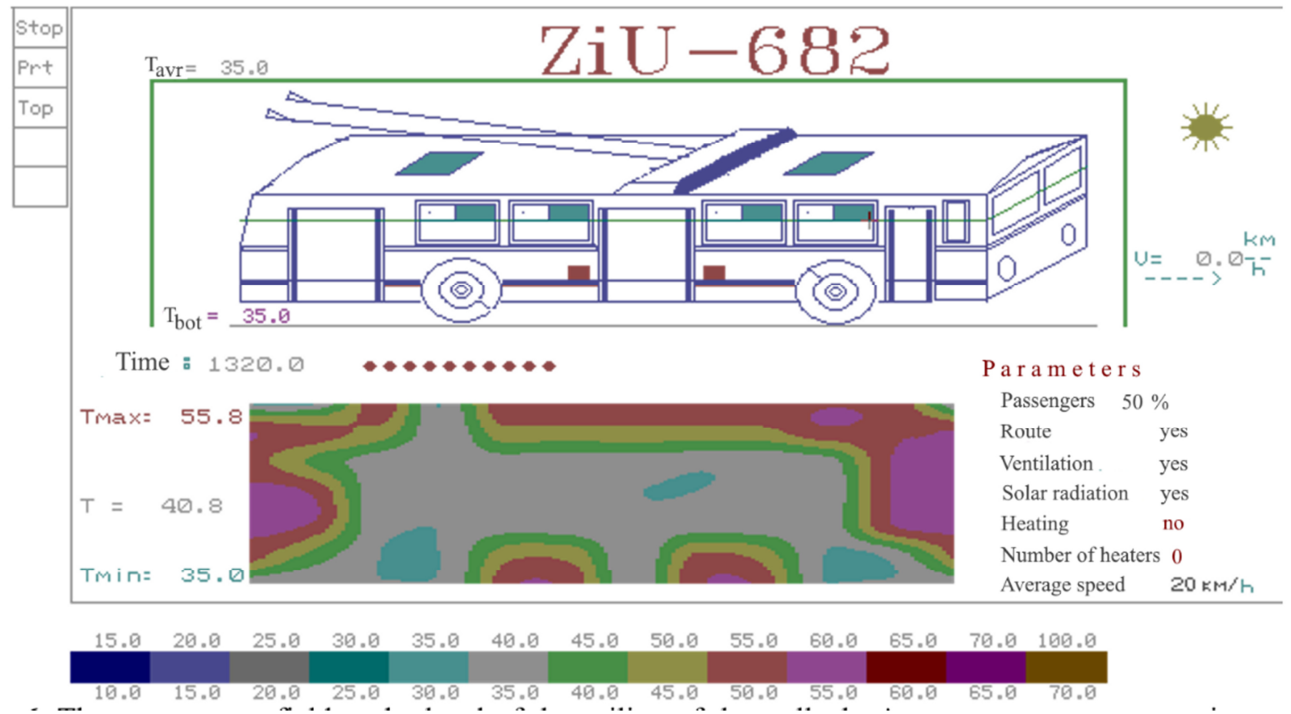

Fig. 6. The temperature field at the level of the ceiling of the trolleybus' passenger compartment in summer. The passenger compartment fullness $100 \%$ 


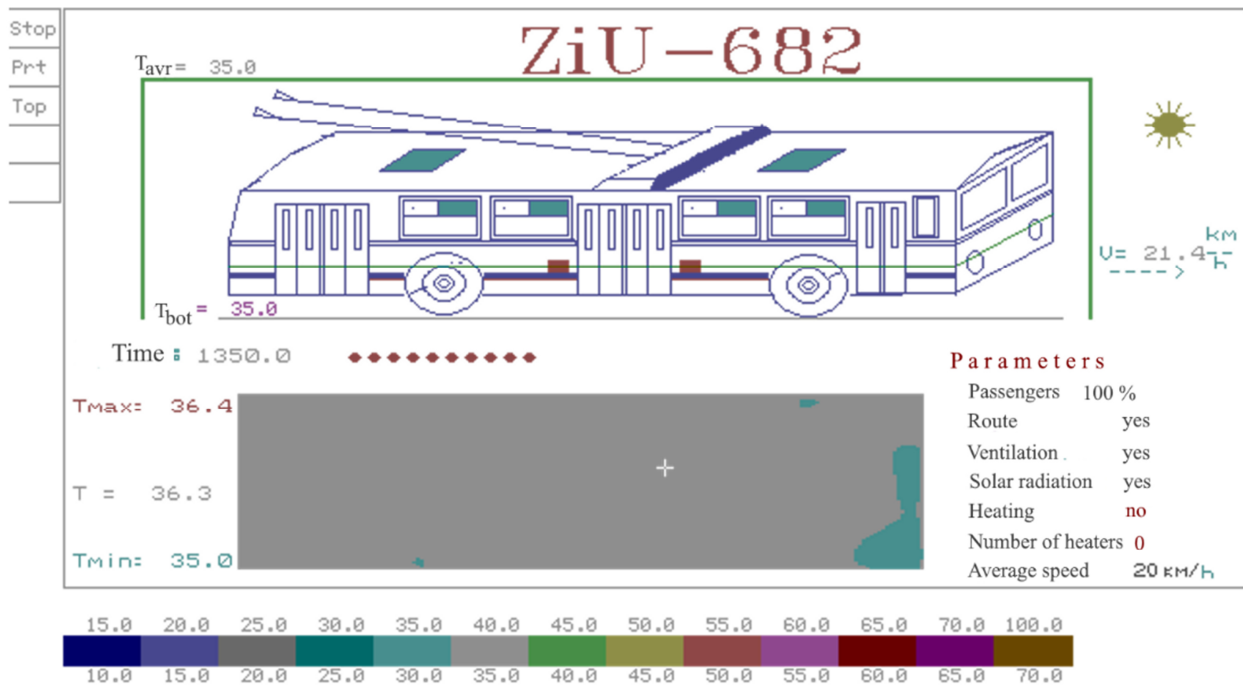

Fig. 7. The temperature field at the level of the sitting places in summer. a) the passenger compartment fullness $100 \%$.

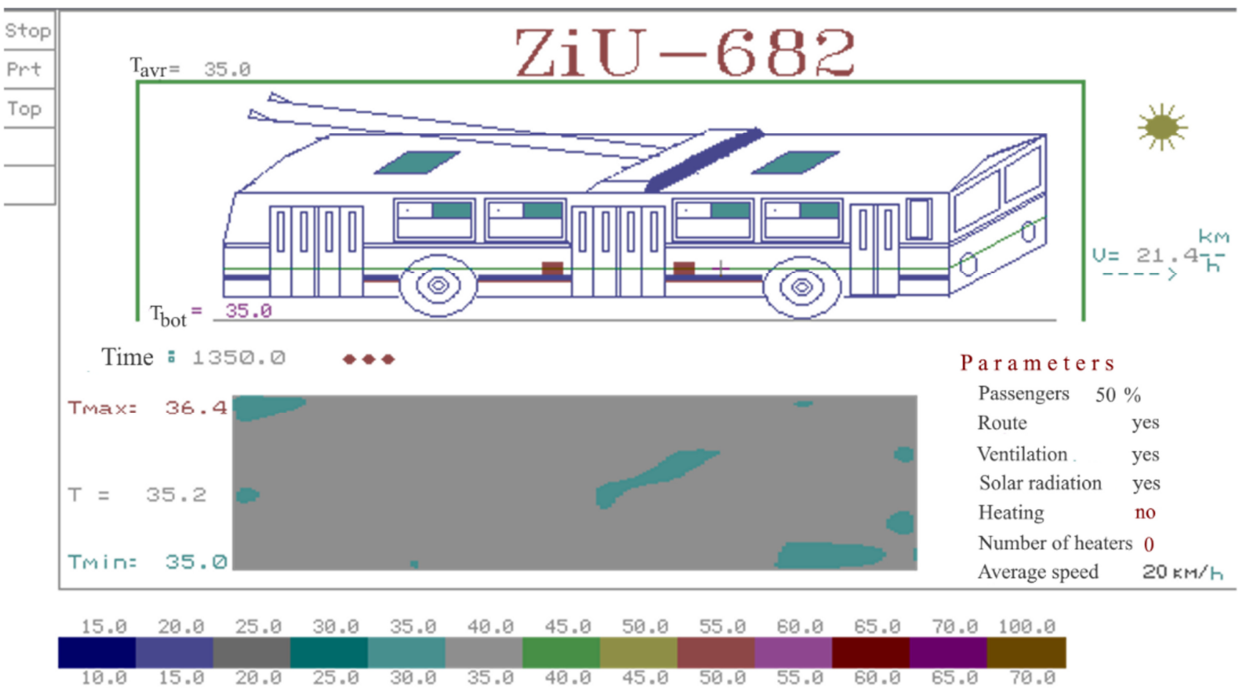

Fig. 8. The temperature field at the level of the sitting places in summer. a) the passenger compartment fullness $50 \%$

In "summer" conditions, non-comfortable temperature takes place in the glazing zone due to solar radiation and internal heat sources of the trolleybus. In the area of the floor, the temperature stays almost equal to the ambient temperature with a uniform distribution throughout the compartment.

If the passenger compartment is filled by $50 \%$ (Fig. 2, Fig. 4, Fig. 6, Fig. 8), the maximum temperature is less than in case when the compartment is filled by $100 \%$ (Fig. 1, Fig. 3, Fig. 5, Fig. 7). Also, the configurations of the temperature fields are slightly different when the compartment fullness is $50 \%$ or $100 \%$, due to a corresponding change in the parameters of convective heat and mass transfer and a decrease in heat generation from the passengers. 


\section{Conclusion}

The constructed mathematical model and its implementation in special software allow the conduction of the automated calculations of nonstationary temperature fields in the trolleybus' passenger compartment with the ability to simulate environmental conditions, cabin ventilation, passenger occupancy, evaluate the effectiveness of heaters in the case of cold seasons, and so on.

Using the proposed methods in the development of transport compartments makes the determination of the quantity and power of heat sources, the location of heat sources and drains, and to introduce the necessary thermal control system, etc. be possible at the design stage. And this will allow maintaining the most comfortable temperature for transported objects - passengers, food, animals, chemicals, etc. with the lowest level of resources spent on this.

The research was carried out within the state assignment of the Ministry of Science and Higher Education of the Russian Federation (theme No. AAAA-A18-118042790040-0)

\section{References}

1. A. V. Golikov, V. M. Pankratov, M. V. Efremov, Gyroscopy and Navigation, 9, 116$123(2018)$

2. V. E. Dzhashitov, V. M Pankratov, The Sensors, devices and systems of aerospace and marine instrument-making in conditions of thermal effects, (St. Petersburg: "Electropribor", 2005)

3. M. A. Barulina, A. V. Golikov, V. M. Pankratov, M. V. Efremov, Scientific instrumentation, 28, 14-23 (2018)

4. M. Barulina, A. Golikov, International Multidisciplinary Scientific GeoConferenceSGEM, 6.2, 691-698 (2019)

5. M. A. Mikheev, I. M. Mikheeva, Fundamentals of heat transfer, (Moscow: Energia, 1977)

6. A. U. Mentsiev, I. A. Mutaev, IOP Conf. Ser.: Mater. Sci. Eng., 734, 012212 (2020)

7. A. V. Delkov, A. A. Kishkin, N. A. Lavrov, et al, Chem Petrol Eng, 51, 714-719 (2016)

8. V. da Silva Rosa, D. de Moraes Jr., Review Heat Transfer of Non-Newtonian Fluids in Agitated Tanks, Heat and Mass Transfer - Advances in Science and Technology Applications, (IntechOpen, 2019)

9. Theodore L. Bergman, et al. Fundamentals of heat and mass transfer, 1048 (2011)

10. A. Golikov, V. Pankratov, M. Barulina, M. Efremov, International Multidisciplinary Scientific GeoConference-SGEM, 6.1, 489-496 (2019) 